\title{
Procalcitonin for Antibiotic Prescription in Chronic Obstructive Pulmonary Disease Exacerbations: Systematic Review, Meta-Analysis, and Clinical Perspective
}

\author{
Ken Chen - Katherine A. Pleasants - Roy A. Pleasants • \\ Tatsiana Beiko · Ronald G. Washburn · Zhiheng Yu • Suodi Zhai • \\ M. Bradley Drummond
}

Received: May 13, 2020 / Published online: July 16, 2020

(C) The Author(s) 2020

\section{ABSTRACT}

The 2020 Global Initiative for Obstructive Lung Disease report indicates that the blood biomarker procalcitonin (PCT) may assist in decision-making regarding the initiation of antibiotics for chronic obstructive pulmonary

Digital Features To view digital features for this article, go to https://doi.org/10.6084/m9.figshare. 12582098 .

Electronic supplementary material The online version of this article (https://doi.org/10.1007/s41030020-00123-8) contains supplementary material, which is available to authorized users.

K. Chen

College of Pharmacy, University of Nebraska

Medical Center, Omaha, NE, USA

K. A. Pleasants · R. G. Washburn

Ralph H. Johnson Veterans Administration Medical Center, Charleston, SC, USA

R. A. Pleasants $(\varangle) \cdot$ M. B. Drummond Division of Pulmonary Diseases and Critical Care Medicine, Department of Medicine, University of North Carolina at Chapel Hill, Chapel Hill, NC, USA e-mail:pleas005@email.unc.edu

\section{T. Beiko}

Division of Pulmonary, Critical Care, Allergy and Sleep Medicine, Department of Medicine, Medical University of South Carolina, Charleston, SC, USA

Z. Yu $\cdot$ S. Zhai

Department of Pharmacy, Peking University Third Hospital, Beijing, China disease (COPD) exacerbations. PCT is an acutephase reactant that increases in response to inflammation and infection, and has been studied in various bacterial infections for initiation and de-escalation of antibacterials. The purpose of this systematic review and metaanalysis was to evaluate the strength of the data on the use of PCT to guide antibiotic prescription in COPD exacerbations. Among the randomized clinical trials included in our metaanalysis, almost all of which were conducted exclusively in the hospital setting. PCT was found to decrease overall antibiotic exposure in COPD exacerbations by 2.01 days $(p=0.04)$, while no apparent effects were found on clinical outcomes (length of hospital stay, $p=0.88$; treatment failure $p=0.51$; all-cause mortality $p=0.28$ ). However, the majority of blood PCT levels in COPD exacerbations were below the manufacturer-recommended cutoff for antibiotics, and the use of this marker was associated with worse outcomes in the intensive care setting. Further, based on additional sensitivity analysis excluding studies with high risk of bias or with converted outcome value, the effect of PCT on antibiotic duration in RCTs was no longer significant $(\mathrm{MD}=-1.88$ days, $95 \% \mathrm{CI}$ $[-3.95,0.19]$ days, $p=0.08$, and $\mathrm{MD}=-1.72$ days, $95 \%$ CI $[-4.28,0.83]$ days, $p=0.19$, respectively). Our review and analysis does not support the use of PCT to guide antibiotic prescription in COPD exacerbations. 
Keywords: Chronic obstructive pulmonary disease; Exacerbation; GOLD report; Procalcitonin

\section{Key Summary Points}

The 2020 Global Initiative for Obstructive Lung Disease report indicates that the blood biomarker procalcitonin (PCT) may assist in guiding the initiation of antibiotics for chronic obstructive pulmonary disease (COPD) exacerbations.

The purpose of this study was to evaluate the strength of data for PCT to guide the prescription of antibiotics in COPD exacerbations. Our study shows that after excluding studies with high risk of bias, the PCT-based protocol may not significantly reduce the duration of antibiotic treatment.

The PCT-based protocol may increase the length of hospital stay for intensive care unit (ICU) patients. PCT has limited value in guiding antibiotic use in COPD exacerbation.

\section{INTRODUCTION}

Chronic obstructive pulmonary disease (COPD) exacerbations are common events that contribute to disease progression, increase healthcare utilization, and lead to the use of drug therapies with potentially significant adverse effects [1]. Although many COPD exacerbations are non-bacterial, and the quality of evidence for antibiotic use is low to moderate in most patient care settings, the 2020 Global Initiative for Chronic Obstructive Lung Disease (GOLD) report recommends treatment with antibacterials in patients who present with sufficient clinical symptoms [1]. Antibacterials can be overprescribed in COPD exacerbations due to confounding factors such as bacterial colonization, unavailability of sputum cultures, and overlap with similarly presenting illnesses. To further streamline antibiotic prescribing in COPD, sensitive and specific clinical biomarkers are needed to guide antibiotic use in COPD exacerbation.

The 2020 GOLD report defines a biomarker as "characteristics that are objectively measured and evaluated as an indicator of normal biologic or pathogenic processes or pharmacological responses to therapeutic interventions" [1]. A number of biological markers have been studied as an adjunct to clinical decision-making to improve antibiotic utilization for lower respiratory tract infections (LRTIs) including COPD. These include blood-based biomarkers such as the acute-phase reactants serum procalcitonin (PCT) and C-reactive protein (CRP), as well as sputum markers such as interleukin-1B. CRP measurement is not routinely recommended by the 2020 GOLD report, due to the fact that elevated levels are present in both viral and bacterial infections [2, 3], and in light of conflicting study results [1] and the need for additional studies. In contrast, the 2020 GOLD report indicates PCT is a potentially useful biomarker for COPD exacerbation because it is more sensitive for bacterial infection than CRP. The GOLD report also notes low to moderate evidence based on two prior meta-analyses [4, 5] for PCT in COPD exacerbation, conflicting findings among studies, and higher mortality when employed in the intensive care unit (ICU) setting [6]. The United Kingdom National Institute for Health and Care Excellence (NICE) guidelines do not provide any recommendations for CRP or PCT in COPD exacerbation [7].

PCT is a peptide precursor of calcitonin and is also an acute-phase reactant in the inflammatory cascade [8]. It is normally produced in low quantities by the thyroid gland; however, in the presence of bacterial infections, synthesis is dramatically increased in all parenchymal tissues including the intestine, liver, and kidney [8]. Such parenchymal tissues lack the ability to convert PCT to calcitonin, resulting in PCT entering the systemic circulation and increasing blood levels. PCT may contribute to tissue damage, but also has anti-inflammatory effects as shown by endotoxin exposure studies in humans $[8,9]$. 
In the patient care setting, serum PCT is measured and then applied clinically based on algorithms that use different thresholds to guide antibacterial initiation or for early discontinuation. The United States (US) Food and Drug Administration (FDA)-approved thresholds for these assays are as follows: antibiotics strongly discouraged if $\mathrm{PCT}<0.1 \mu \mathrm{g} \cdot \mathrm{L}^{-1}$, discouraged for serum levels between 0.1 and $0.25 \mu \mathrm{g} \cdot \mathrm{L}^{-1}$, recommended for levels $>0.25$ to $0.5 \mu \mathrm{g} \cdot \mathrm{L}^{-1}$, and strongly recommended for levels $>0.5 \mu \mathrm{g} \cdot \mathrm{L}^{-1}$.

To better inform clinicians on the current use of PCT for antibiotic treatment of COPD exacerbations, we undertook a systematic review and meta-analysis to investigate the impact of a PCT-based protocol on antibiotic prescription and clinical outcomes in patients with COPD exacerbations.

\section{METHODS}

PubMed, EmBase, the Cochrane Library, and ClinicalTrials.gov were searched through February 29, 2020. The search terms were the combination of subject terms and free terms. Details of the search terms for PubMed are shown in Supplementary Appendix 1. Studies on the use of PCT-based protocols for guiding antibiotic use in patients with COPD exacerbations were eligible for inclusion. Diagnosis of COPD exacerbations was mandatory. No restrictions were applied to patient care settings, details of PCT-based protocols, follow-up time, study design, or availability of full texts. Only studies in English or Chinese were included. The primary study outcome was the length of antibiotics therapy, while secondary outcomes included hospital length of stay (LOS), treatment failure, all-cause and respiratory-related mortality, and all-cause and respiratory-related readmission. Eligible studies had to report at least one study outcome. Two reviewers (KC and $\mathrm{ZY}$ ) independently screened titles and abstracts of retrieved citations initially, looked into full texts, and identified final studies.

Two reviewers (KC and ZY) also independently extracted data from original studies and performed a quality appraisal. The following data were extracted from identified studies: study design, patient characteristics, PCT-based protocol, sample size, outcome values, followup time, definition of treatment failure, etc. For continuous outcomes, median and interquartile were converted into mean and standard deviation, respectively [10].

The Cochrane risk of bias tool was applied to evaluate the risk of bias of included randomized controlled trials (RCTs) [11]. The NewcastleOttawa scale (NOS) was applied to evaluate the risk of bias of the included observational studies [12]. Because we judged outcomes were not likely to be influenced by lack of blinding of participants and personnel, the item was rated as "low risk of bias" for all RCTs. We required PCT levels not available to providers of the standard therapy group. If not, the item "other risk of bias" would be judged high for RCTs. When evaluating quality of observational studies, predicted forced expiratory volume in the first second (FEV1)\% or GOLD stage was considered the most important baseline characteristic when evaluating comparability between cohorts, while ICU admission, comorbidity, and age were considered less important. Quality of evidence for each outcome was assessed using the Grading of Recommendations Assessment, Development and Evaluation (GRADE) method. Publication bias was evaluated only if more than 10 studies were included in a metaanalysis.

Meta-analysis was performed using RevMan 5.1 (Cochrane Information Management System). The Mantel-Haenszel and inverse variance methods were used as the statistical model to calculate the risk ratio (RR) and mean difference (MD) for binary and continuous outcomes, respectively. The Cochran $\mathrm{Q} \chi^{2}$ test and $I^{2}$ statistic were used to assess heterogeneity among studies. A value of $p<0.1$ was considered significant because of the low statistical power of the $\chi^{2}$ test for heterogeneity. A random effects model was applied if $p<0.1, I^{2}>50 \%$, or clinical heterogeneity was observed among studies; otherwise, a fixed effects model was applied.

Subgroup analysis was performed based on no ICU admission, PCT level cutoff $\left(0.25 \mu \mathrm{g} \cdot \mathrm{L}^{-1}\right.$ vs. $\left.<0.25 \mu \mathrm{g} \cdot \mathrm{L}^{-1}\right), \quad$ and follow-up time 
( $\leq 1$ month vs. $>1$ month, for mortality and readmission outcomes only), and sensitivity analysis was performed based on the risk of bias of included studies, existence of continuous data converted from median and/or interquartile, and baseline antibiotics upon admission.

This article is based on previously conducted studies and does not contain any studies with human participants or animals performed by any of the authors.

\section{RESULTS OF SYSTEMATIC REVIEW AND META-ANALYSIS}

\section{Overview of Studies}

A total of 14 studies were included in the metaanalysis of PCT as a biomarker for COPD exacerbation (Fig. 1) [6, 13-25]. Reasons for excluding studies are summarized in Supplementary Appendix 2; the most common reason for excluding a study was the lack of reported data specifically on COPD exacerbation. Characteristics of the included studies are shown in Supplementary Appendix 3. Raw data are shown in Supplementary Appendix 4. The types of patients and settings varied among the studies in COPD exacerbation. Only two studies reported that subjects were classified as type I or II Anthonisen exacerbation (Supplementary Appendix 3). All but two of the studies of PCT in COPD exacerbation were conducted in the hospital setting [13-15, 17, 18, 20-23, 25], while fewer studies were undertaken in the emergency department $[16,19]$ or ICU setting $[6,24]$, and none in the outpatient setting.

Target threshold PCT blood levels varied among studies, where nine studies utilized $0.25 \mu \mathrm{g} \cdot \mathrm{L}^{-1}[13-19,22,24]$, consistent with US FDA recommendations. Daubin et al. and Stolz et al. used lower PCT level cutoffs $(0.1$ and $0.1-0.25 \mu \mathrm{g} \cdot \mathrm{L}^{-1}$, respectively) $[6,20]$. An important finding among these studies is that more than three-fourths of patients had PCT blood levels below the $0.25 \mu \mathrm{g} \cdot \mathrm{L}^{-1}$ threshold recommended by the FDA for antibiotic prescription $[13,18,20]$. There was a wide variance in mean PCT blood levels in COPD exacerbation ranging from 0.06 to $1.44 \mu \mathrm{g} \cdot \mathrm{L}^{-1} \quad$ (Supplementary
Appendix 3); some of this variation is related to higher PCT levels reported with pneumonia.

\section{Quality Appraisal of Clinical Studies of Procalcitonin in COPD Exacerbation}

The risk of bias among included RCTs and observational studies is shown in Fig. 2 and Supplementary Appendix 5, respectively. The GRADE analysis for all outcomes is summarized in Table 1.

For RCT studies, risk of bias was not assessed for two studies with abstract only due to lack of information [22, 24]. Because further information was provided by the authors of one study, we assessed its risk of bias [23]. Only two out of nine studies reached a full score by the risk of bias assessment $[16,19]$. Four studies did not blind outcome assessors [14, 15, 17, 21], while two studies were judged as high risk for selection $[15,17]$ and attrition bias $[17,21]$, respectively. We could not confirm whether PCT results were accessible to providers of the standard-therapy group in 4 studies $[14,15,17,23]$.

For observational studies, three studies used a before-after study design, and appropriate selection of a non-exposed group was unclear $[13,18,25]$. The existence of comorbidity was not comparable between exposed and non-exposed groups in one study [18]. We were unsure about the comparability between their exposed and non-exposed groups for two studies [13, 25].

\section{PCT-Based Protocol and Length of Antibiotic Treatment}

A total of six RCTs $[6,14-16,19,23]$ and two observational studies $[13,25]$ reported length of antibiotic treatment. Outcome measures of three studies were converted from median and/ or interquartile $[15,19,25]$. A random effects model was applied to the meta-analysis of RCTs. Results from both RCTs and observational studies showed that the use of PCT-based protocols significantly reduced the length of antibiotic treatment in COPD exacerbation $(\mathrm{MD}=-2.01$ days, 95\% confidence interval $(\mathrm{CI})$ $[-3.89,-0.14]$ days, $p=0.04$, moderate quality, and $\mathrm{MD}=-1.64$ days, $95 \%$ CI $[-2.91,-0.36]$ 




Fig. 1 PRISMA flow diagram of study screening

days, $p=0.01$, very low quality for RCTs and observational study, respectively) (Fig. 3).

Of the six RCTs, only Daubin et al. was conducted in an ICU setting and utilized a lower PCT level cutoff $\left(0.1 \mu \mathrm{g} \cdot \mathrm{L}^{-1}\right)$. In this study, patients who used PCT-based protocols had similar antibiotic treatment duration $(\mathrm{MD}=0.20$ days, 95\% CI $[-1.37,1.77]$ days, $p=0.80)$ compared with the standard therapy group. A subgroup difference test between Daubin et al. and the other five RCTs was positive $(p=0.05)$ [6]. After excluding Nangia et al. [23], who did not report a PCT level cutoff, no significant difference was found between Daubin et al. and the other four RCTs. Since both observational studies showed a significant reduction in the length of antibiotic treatment, there was no need to conduct subgroup analysis on the observational studies.

\section{PCT-Based Protocol and Length of Hospital Stay}

A total of nine RCTs [6, 14-17, 19-21, 23] and three observational studies $[13,18,25]$ reported hospital LOS. Outcome measures of five studies 


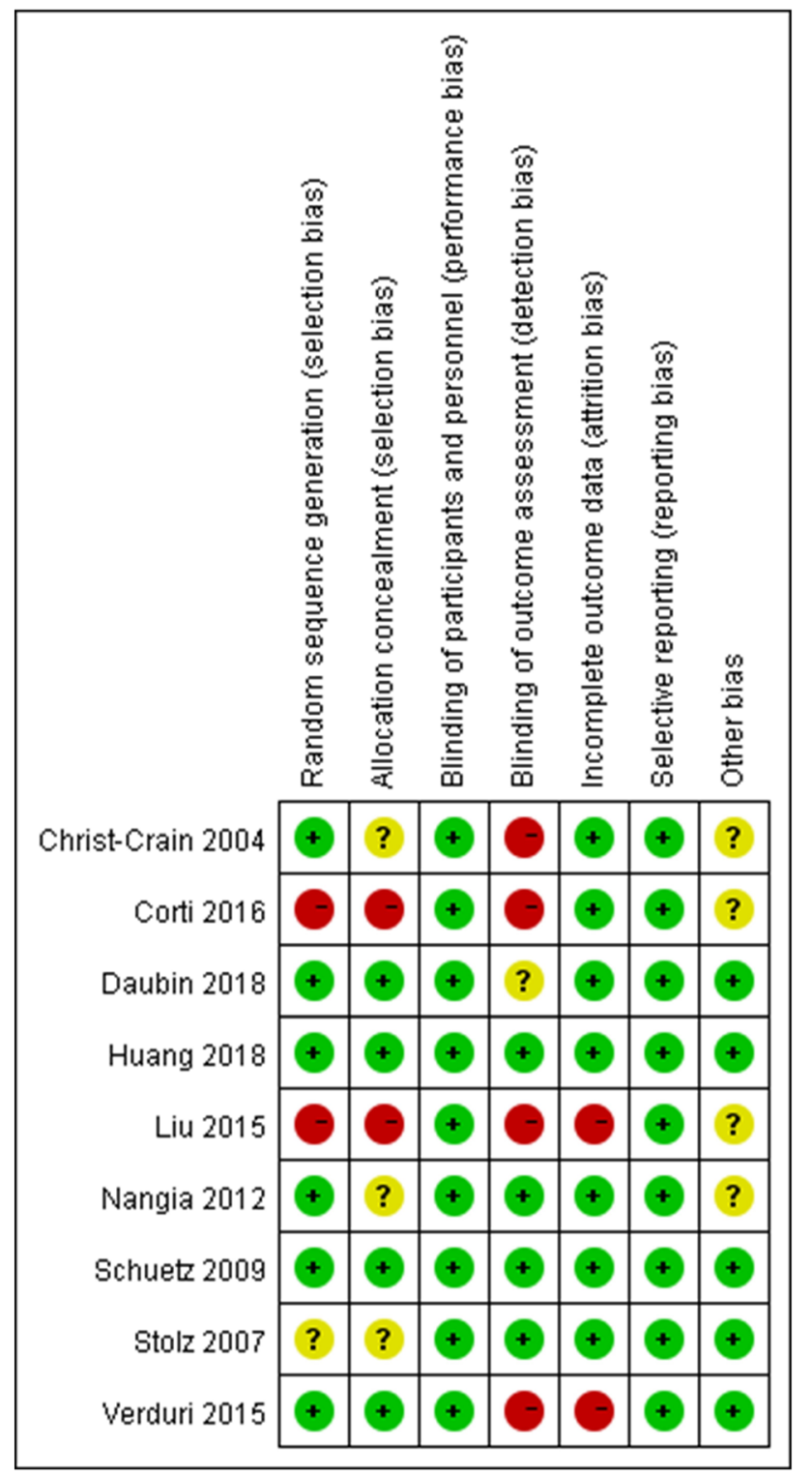

Fig. 2 Risk of bias of included randomized controlled trials for PCT in COPD exacerbations

were converted from median and/or interquartile $[15,19-21,25]$. A random effects model was applied to the meta-analysis of RCTs, and showed that among patients with PCT-based protocols, the LOS was similar to that in patients managed by standard therapy (MD $=0.06$ days, 95\% CI $[-0.71,0.83]$ days, $p=0.88$, low quality). For observational studies, the use of PCT-based protocols had no effect on LOS compared to standard management when a random effects model was applied to the meta- analysis $\quad(\mathrm{MD}=-0.17$ days, $95 \%$ CI $[-1.62$, 1.28 ] days, $p=0.82$, very low quality) (Supplementary Appendix 6).

Subgroup analysis could only be performed in RCTs due to limited data. Unlike non-ICU patients, ICU patients following a PCT-based protocol had significantly longer hospital LOS compared with the standard therapy group $(\mathrm{MD}=4.03$ days, $95 \%$ CI $[0.74,7.32]$ days, $p=0.02$ ) [6]. A subgroup difference test between ICU and non-ICU patients was positive 


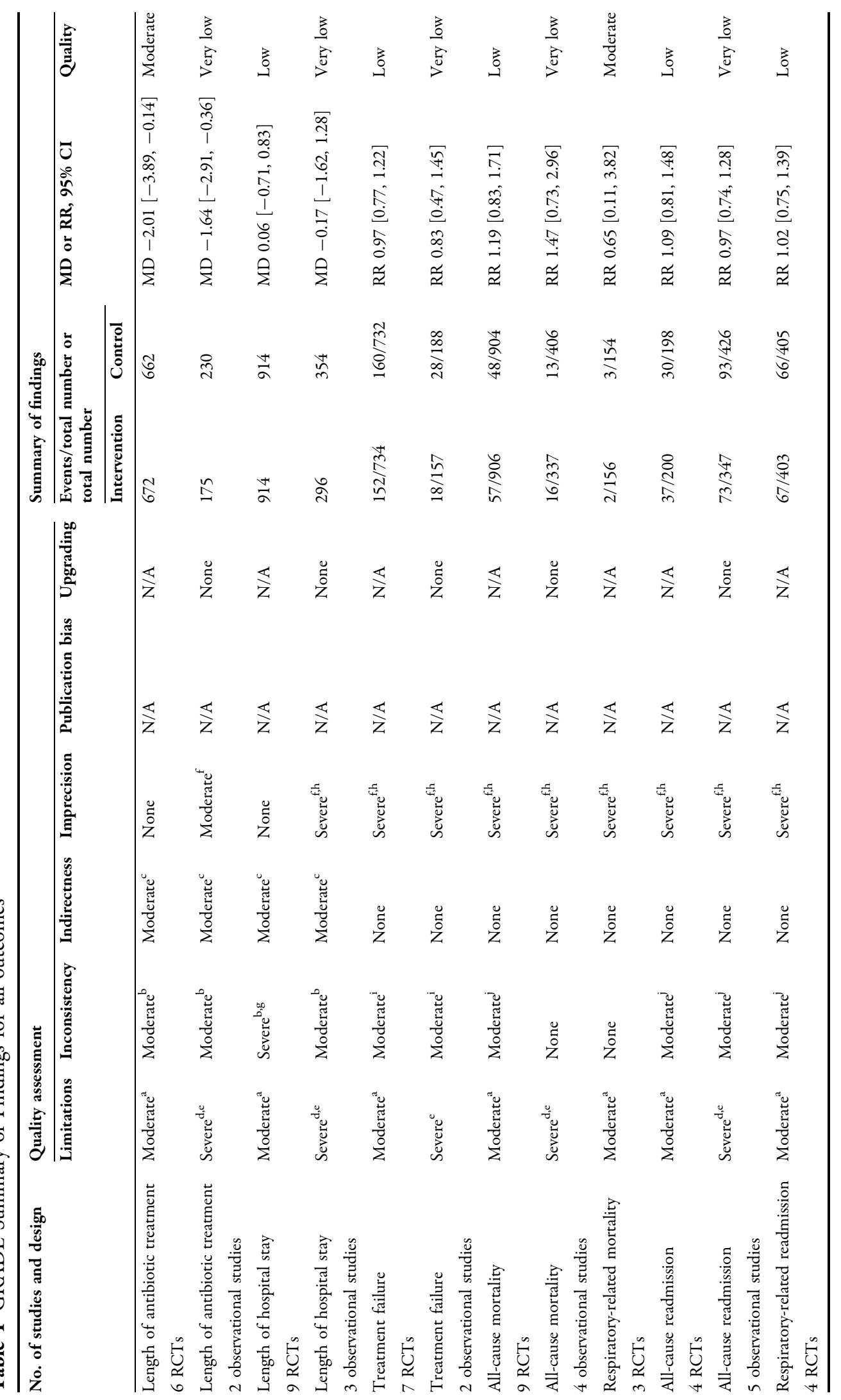




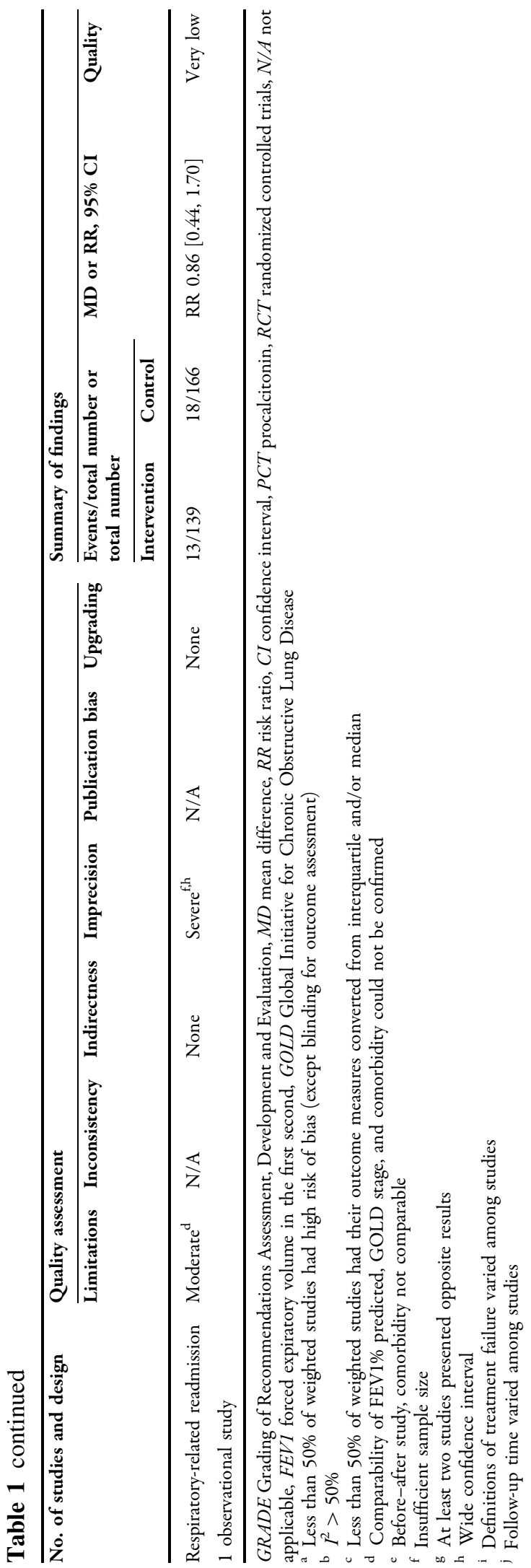

$(p=0.02)$. Subgroup analysis on PCT level cutoff did not show significant results.

\section{PCT-Based Protocol and Treatment Failure}

A total of seven RCTs [15-17, 19-21, 23] and two observational studies [18, 25] reported treatment failure. Criteria of treatment failure in most studies included worsening symptoms, ICU admission, death, and readmission within $72 \mathrm{~h}$ to 30 days of admission. Since definitions of treatment failure varied among studies, a random effects model was applied to the metaanalysis of RCTs. Results from both the RCTs and the observational study showed that COPD patients with exacerbation managed with PCTbased protocols had similar treatment failure rates as the standard therapy group $(\mathrm{RR}=0.97$, $95 \%$ CI $[0.77,1.22], p=0.27$, low quality, and $\mathrm{RR}=0.83,95 \%$ CI $[0.47,1.45], p=0.81$, very low quality for RCTs and observational studies, respectively) (Supplementary Appendix 6). Subgroup analysis on PCT level cutoff did not show any significant results.

\section{PCT-Based Protocol and Mortality}

A total of 13 studies (nine RCTs $[6,14-17,19-21,23]$ and four observational studies $[13,18,24,25]$ ) and four studies (three RCTs $[15,17,23]$ and one observational study [13]) reported all-cause and respiratory-related mortality within 21 days to 6 months, respectively. A fixed effects model was applied to the meta-analysis of both RCTs and observational studies. No respiratory-related death occurred in patients from the observational study. Results from both the RCTs and the observational study showed that PCT-based and standard of carebased protocols had similar all-cause mortality $(\mathrm{RR}=1.19,95 \% \mathrm{CI}[0.83,1.71], p=0.74$, low quality, and $\mathrm{RR}=1.47,95 \% \mathrm{CI}[0.73,2.96]$, $p=0.28$, very low quality for RCTs and observational studies, respectively) and respiratoryrelated mortality rates compared with the standard therapy group $(\mathrm{RR}=0.65,95 \% \mathrm{CI}$ $[0.11,3.82], p=0.63$, moderate quality for RCTs) (Supplementary Appendix 6). 


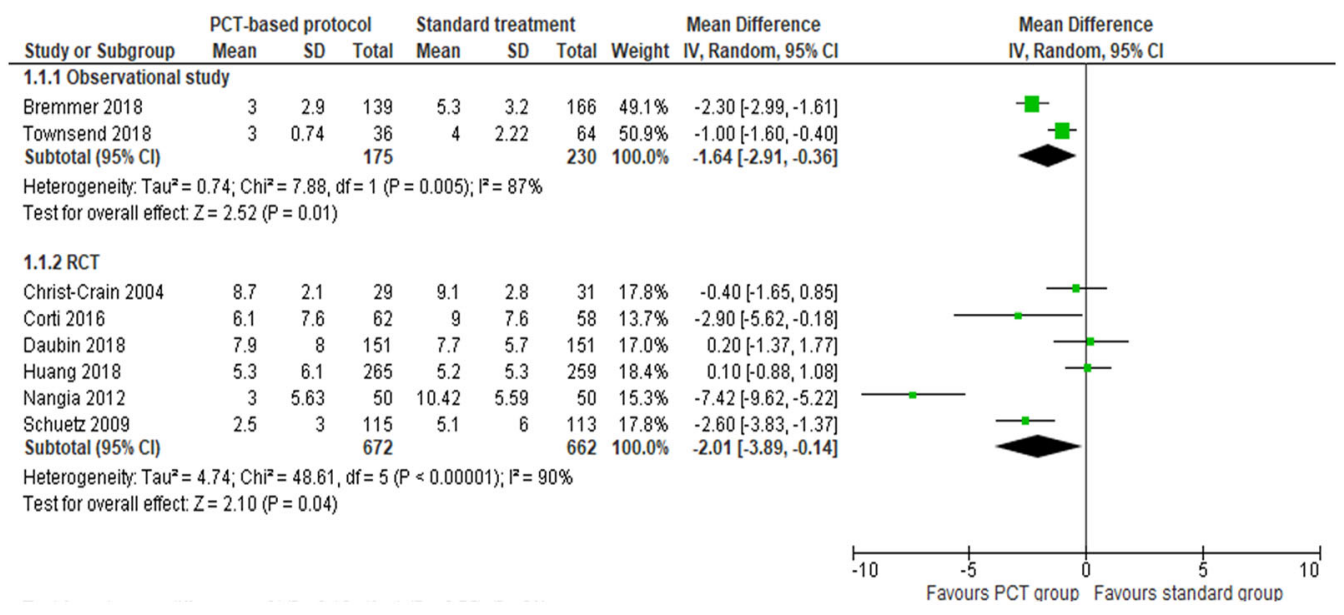

Fig. 3 Forest plot of the effects of PCT-based protocol versus standard treatment on length of antibiotic treatment

Due to limited data, no subgroup analysis was performed on respiratory-related mortality. Subgroup analysis on ICU admission, PCT level cutoff, or follow-up time did not show any significant results.

\section{PCT-Based Protocol and Hospital Readmission}

A total of nine studies (four RCTs [15, 16, 21, 23] and five observational studies $[13,18,22,24,25])$ and five studies (four RCTs $[6,15,20,21]$ and one observational study [13]) reported all-cause and respiratory-related readmission within 28 days to 6 months, respectively. A fixed effects model was applied to meta-analysis of either study design or outcome. Results from both RCTs and observational study showed that patients managed with PCT-based protocols had similar all-cause readmission $(\mathrm{RR}=1.09,95 \%$ CI $[0.81,1.48]$, $p=0.56$, low quality, and $\mathrm{RR}=0.97,95 \% \mathrm{CI}$ $[0.74,1.28], p=0.84$, very low quality for RCTs and observational studies, respectively) and respiratory-related readmission rates compared with the standard therapy group $(\mathrm{RR}=1.02$, $95 \%$ CI $[0.75,1.39], p=0.91$, low quality, and $\mathrm{RR}=0.86,95 \%$ CI $[0.44,1.70], p=0.67$, very low quality for RCTs and observational study, respectively) (Supplementary Appendix 6).

Of five observational studies reporting allcause readmission, only Sabrine et al. studied
ICU patients and had follow-up time longer than 30 days. In this study, patients who used PCT-based protocols had a significantly lower all-cause readmission rate $(\mathrm{RR}=0.44,95 \% \mathrm{CI}$ $[0.21,0.95], p=0.04)$ compared with the standard therapy group [24]. A subgroup difference test between Sabrine et al. and the other four observational studies was positive $(p=0.03)$. Other subgroup analysis on all-cause and respiratory-related readmission did not show any significant results.

\section{Sensitivity Analysis}

After removal of studies with one or more highrisk-of-bias items (except blinding for outcome assessment) [15] or with converted outcome value $[15,19]$, the difference in the length of antibiotic treatment between the two groups was no longer significant $(\mathrm{MD}=-1.88$ days, $95 \%$ CI $[-3.95,0.19]$ days, $p=0.08$, and $\mathrm{MD}=-1.72$ days, $95 \%$ CI $[-4.28,0.83]$ days, $p=0.19$, respectively) in RCT meta-analysis. Also, after removal of studies with one or more high-risk-of-bias items (except blinding for outcome assessment) $[15,17,21]$ and application of a fixed effects model, the PCT group had a significantly longer hospital LOS compared with the standard therapy group $(\mathrm{MD}=0.56$ days, $95 \%$ CI $[0.06,1.05]$ days, $p=0.03$ ), and the $I^{2}$ was reduced from 65 to $45 \%$. For observational studies, after removal of 
Townsend et al., which had a lower NOS score and a converted outcome value [25], and application of a fixed effects model, the PCT group had a significant 1-day shorter hospital LOS (MD $=-1.01$ days, 95\% CI $[-1.62,-0.40]$ days, $p=0.001$ ) compared with the standard therapy group, and the $I^{2}$ was reduced from 84 to $37 \%$. Our results proved robust to each of the remaining predefined sensitivity analyses.

\section{DISCUSSION}

Our analysis does not support the use of serum PCT measurements to guide antibacterial prescription in COPD exacerbation, as it does little to improve antibiotic use, clinical outcomes, or healthcare utilization. PCT-based protocols did result in a shorter overall duration of antibiotic therapy by a mean of 2 days in the hospital setting; however, our sensitivity analysis using only more well-designed studies indicated that the effect on duration was no longer significant. Treatment failure, hospital readmission, and overall mortality were similar between PCTbased and standard antibiotic treatment. Although no differences were found in overall respiratory and all-cause mortality, two studies conducted in the ICU setting reported higher mortality with the use of PCT, indicating that it should not be used in critically ill patients with COPD exacerbations [6, 24]. Hospital LOS and risk of readmission, key healthcare utilization metrics, were also similar between PCT-guided antibiotic therapy and standard of care.

The FDA approved the use of serum PCT assays as an anti-infective biomarker in the United States in 2012 [26], and then expanded indications in 2017 to include assisting with initiating or discontinuing antibiotics in lower respiratory tract infections and discontinuing antibiotics in sepsis. Among the studies included in the analysis (Table 1), PCT levels were often below the FDA-recommended $0.25 \mu \mathrm{g} \cdot \mathrm{L}^{-1}$ threshold [13-16]. If the FDA-recommended PCT threshold had been utilized, it would have been more likely that antibiotics would have been discouraged, even in patients presenting to the hospital with Anthonisen type I or II exacerbation [14]. An upcoming randomized, double-blind, placebo-controlled study (ABACOPD) targeting 1000 patients with moderate acute COPD exacerbation will use an upper PCT limit of $0.25 \mu \mathrm{g} \cdot \mathrm{L}^{-1}$ to enroll patients in order to avoid the inclusion of patients with possible pneumonia [27].

In addition to our meta-analysis, inherent limitations in PCT measurement for COPD exacerbation should be taken into consideration. Apart from typically low serum levels with COPD exacerbation in the hospital setting, two studies showed that PCT was unreliable in differentiating between a viral and bacterial infection $[2,3]$, and in one study did not differentiate between stable and acutely ill patients [28]. Because PCT alone does not correlate well with the presence of bacteria in COPD exacerbation, recovery without antibiotics or with an abbreviated course was assumed by investigators to equate to the absence of a clinically significant bacterial infection. Finally, PCT levels can be elevated with mycobacterial and systemic candida infections as well as renal disease, cardiogenic shock, and trauma [29].

The Anthonisen exacerbation type is considered the gold standard for determining the need for antibacterials in COPD exacerbation $[1,7]$, and the effectiveness of other infectious biomarkers markers should thus be judged on this basis. In two studies of COPD exacerbation $[21,30]$, PCT serum levels did not differ between purulent and mucoid sputum, and one would expect higher values with the former, as bacteria are more likely to be present. In a placebo-controlled study of doxycycline for COPD type I and II exacerbations, antibiotic treatment resulted in improved efficacy even with mean PCT levels of $0.1 \mu \mathrm{g} \cdot \mathrm{L}^{-1}$, which according to the PCT-based protocol would not have justified the use of antibiotics [31].

Our analysis showed that healthcare utilization and clinical outcomes did not differ between PCT- and standard-of-care-based antibiotic treatment of COPD exacerbations. While the lack of a negative effect on healthcare utilization appears to be a favorable finding for PCT-guided antibiotic treatment, two placebocontrolled studies showed that when employing the Anthonisen exacerbation criteria to prescribe antibiotics, treatment resulted in a 
lower probability of hospital readmission $[31,32]$. Particularly concerning are the worse outcomes reported with the use of PCT for COPD exacerbation in the ICU setting $[6,24]$. In a prospective, randomized, controlled clinical trial of COPD exacerbation in the ICU setting in 89 patients without antibiotic therapy at baseline, Daubin et al. found that the use of PCT significantly increased 3-month mortality [19/ $31(31 \%)$ vs. $7 / 58(12 \%), p=0.015$ ] [6]. Further, a retrospective study of 93 patients with COPD exacerbation who were mechanically ventilated in the ICU found that mortality rates in the PCT-guided group versus control group were $19 \%$ versus $9.5 \%$, respectively $(p=0.17)$ [24].

One potential area where PCT levels might be clinically useful in COPD patients presenting with respiratory symptoms is in differentiating between pneumonia and exacerbation. Among the studies of COPD exacerbation conducted in the hospital setting, we found that all but one reported several-fold higher PCT levels with pneumonic COPD exacerbation. The mean or median was near or above $0.25 \mu \mathrm{g} \cdot \mathrm{L}^{-1}$, compared to values of $0.1 \mu \mathrm{g} \cdot \mathrm{L}^{-1}$ or lower for nonpneumonic exacerbations [33-38]. However, American Thoracic Society/Infectious Disease Society of America guidelines for communityacquired pneumonia recommend antibiotics regardless of the PCT level [39]. This was based in part on the poor sensitivity of PCT to differentiate between viral and bacterial infections. The presence of COPD as a comorbidity was not discussed with that recommendation.

\section{Strengths and Limitations}

The main strengths of this study include the use of a rigorous and up-to-date meta-analysis. Compared to the meta-analyses by Mathioudakis, we include additional RCTs as well as observational studies [4]. A recent meta-analysis by Ni et al., which included many of the studies in our analysis, found that the use of PCT decreased antibiotic use in patients with COPD exacerbation, and suggested that it had moderate ability to distinguish bacterial infection. However, they pooled RCTs and observational studies, which is a less reliable approach $[5,40]$.
We used the GRADE method to evaluate the quality of evidence for each outcome of interest. Neither of these meta-analyses discussed the low PCT levels in COPD exacerbation below the recommended threshold at which antibiotics are encouraged. We also identified a potential patient group that may benefit from additional investigation, specifically a study on the use of PCT testing to distinguish pneumonia from COPD exacerbation.

The principal limitation of this study is the low level of evidence of the clinical studies available for the meta-analysis. No single metaanalysis included more than 10 studies, and therefore publication bias could not be assessed. An additional important limitation is that nearly all studies were conducted in the hospital setting, whereas most COPD flares occur in the outpatient setting. It was apparent that PCT measurement should not be used for COPD exacerbations in the ICU setting. However, we did not investigate the impact of using a PCTbased protocol on the length of ICU stay in patients with COPD exacerbations, which is an important clinical outcome to consider for ICU patients. Most studies looked at clinical outcomes as a secondary, not primary, endpoint. Lastly, although PCT measurements often resulted in decreased antibacterial use, data were not available to assess the impact on antibiotic-related adverse effects.

\section{CONCLUSION}

Our meta-analysis shows that PCT has limited value in guiding antibiotic use in COPD exacerbation. PCT blood levels in such cases are often below the FDA-recommended threshold for initiating antibacterial treatment, even with type I or II exacerbations requiring hospitalization, a setting where antibiotic efficacy has been demonstrated. In particular, PCT should not be used in the ICU setting to guide antibacterial prescribing in COPD exacerbation. Further study may be warranted on the ability of PCT to differentiate pneumonia from exacerbation and on randomized PCT use based on Anthonisen exacerbation criteria, particularly with regard to sputum purulence. 


\section{ACKNOWLEDGEMENTS}

Funding. Ken Chen is sponsored by the China Scholarship Council. Otherwise, no funding or sponsorship was received for this study or publication of this article.

Authorship. All named authors meet the International Committee of Medical Journal Editors (ICMJE) criteria for authorship for this article, take responsibility for the integrity of the work as a whole, and have given their approval for this version to be published.

Disclosures. M. Bradley Drummond reports personal fees from Boehringer-Ingelheim, personal fees from GlaxoSmithKline, personal fees from AstraZeneca, personal fees from MylanTheravance, grants from the Department of Defense, personal fees from Novavax, personal fees from Parion, personal fees from Midmark, personal fees from Philips, and grants from NIHNHLBI, outside the submitted work. All other authors (Ken Chen, Katherine A. Pleasants, Roy A. Pleasants, Tatsiana Beiko, Ronald G. Washburn, Zhiheng $\mathrm{Yu}$, Suodi Zhai) have nothing to disclose.

Compliance with Ethics Guidelines. This article is based on previously conducted studies and does not contain any studies with human participants or animals performed by any of the authors.

Data Availability. All data generated or analyzed during this study are included in this published article/as supplementary information files.

Open Access. This article is licensed under a Creative Commons Attribution-NonCommercial 4.0 International License, which permits any non-commercial use, sharing, adaptation, distribution and reproduction in any medium or format, as long as you give appropriate credit to the original author(s) and the source, provide a link to the Creative Commons licence, and indicate if changes were made. The images or other third party material in this article are included in the article's Creative Commons licence, unless indicated otherwise in a credit line to the material. If material is not included in the article's Creative Commons licence and your intended use is not permitted by statutory regulation or exceeds the permitted use, you will need to obtain permission directly from the copyright holder. To view a copy of this licence, visit http://creativecommons.org/licenses/by$\mathrm{nc} / 4.0 /$.

\section{REFERENCES}

1. Global Initiative for Chronic Obstructive Lung Disease Inc. Global strategy for the diagnosis, management and prevention of chronic obstructive pulmonary disease 2020 report. 2020. https://www. goldcopd.org.

2. Chang $\mathrm{CH}$, Tsao $\mathrm{KC}, \mathrm{Hu} \mathrm{HC}$, et al. Procalcitonin and C-reactive protein cannot differentiate bacterial or viral infection in COPD exacerbation requiring emergency department visits. Int J Chron Obstruct Pulmon Dis. 2015;10:767-74.

3. Clark TW, Medina MJ, Batham S, et al. C-reactive protein level and microbial aetiology in patients hospitalised with acute exacerbation of COPD. Eur Respir J. 2015;45(1):76-86.

4. Mathioudakis AG, Chatzimavridou-Grigoriadou V, Corlateanu A, et al. Procalcitonin to guide antibiotic administration in COPD exacerbations: a metaanalysis. Eur Respir Rev. 2017;26(143):160073.

5. Ni W, Bao J, Yang D, et al. Potential of serum procalcitonin in predicting bacterial exacerbation and guiding antibiotic administration in severe COPD exacerbations: a systematic review and meta-analysis. Infect Dis (Lond). 2019;51(9):639-50.

6. Daubin C, Valette X, Thiolliere F, et al. Procalcitonin algorithm to guide initial antibiotic therapy in acute exacerbations of COPD admitted to the ICU: a randomized multicenter study. Intensive Care Med. 2018;44(4):428-37.

7. National Institute for Health and Care Excellence. Chronic obstructive pulmonary disease in over 16s: diagnosis and management. 2018. https://www. nice.org.uk/guidance/ng115.

8. Schneider HG, Lam QT. Procalcitonin for the clinical laboratory: a review. Pathology. 2007;39(4): 383-90. 
9. Meisner M. Update on procalcitonin measurements. Ann Lab Med. 2014;34(4):263-73.

10. Wan X, Wang W, Liu J, et al. Estimating the sample mean and standard deviation from the sample size, median, range and/or interquartile range. BMC Med Res Methodol. 2014;14:135.

11. Higgins J, Green S. Cochrane Handbook for Systematic Reviews of Interventions. 2019. Available from: http://handbook.cochrane.org.

12. Wells GA, Shea B, O'Connell D et al. The NewcastleOttawa Scale (NOS) for assessing the quality of nonrandomised studies in meta-analyses. 2019. Available from: http://www.ohri.ca/programs/ clinical_epidemiology/oxford.asp.

13. Bremmer DN, DiSilvio BE, Hammer C, et al. Impact of procalcitonin guidance on management of adults hospitalized with chronic obstructive pulmonary disease exacerbations. J Gen Intern Med. 2018;33(5):692-7.

14. Christ-Crain M, Jaccard-Stolz D, Bingisser R, et al. Effect of procalcitonin-guided treatment on antibiotic use and outcome in lower respiratory tract infections: cluster-randomised, single-blinded intervention trial. Lancet. 2004;363(9409):600-7.

15. Corti C, Fally M, Fabricius-Bjerre A, et al. Point-ofcare procalcitonin test to reduce antibiotic exposure in patients hospitalized with acute exacerbation of COPD. Int J Chron Obstruct Pulmon Dis. 2016;11: 1381-9.

16. Huang DT, Yealy DM, Filbin MR, et al. Procalcitonin-guided use of antibiotics for lower respiratory tract infection. N Engl J Med. 2018;379(3):236-49.

17. Liu SS, Zhang YB. The value of serum procalcitonin level in guiding the use of antibiotic in patients with acute exacerbation of chronic obstructive pulmonary disease. Chin J Antibiot. 2015;40(6): 459-63.

18. Picart J, Moiton MP, Gauzere BA, et al. Introduction of a PCT-based algorithm to guide antibiotic prescription in COPD exacerbation. Med Mal Infect. 2016;46(8):429-35.

19. Schuetz P, Christ-Crain M, Thomann R, et al. Effect of procalcitonin-based guidelines vs standard guidelines on antibiotic use in lower respiratory tract infections: the ProHOSP randomized controlled trial. JAMA. 2009;302(10):1059-66.

20. Stolz D, Christ-Crain M, Bingisser R, et al. Antibiotic treatment of exacerbations of COPD: a randomized, controlled trial comparing procalcitoninguidance with standard therapy. Chest. 2007;131(1):9-19.
21. Verduri A, Luppi F, D'Amico R, et al. Antibiotic treatment of severe exacerbations of chronic obstructive pulmonary disease with procalcitonin: a randomized noninferiority trial. PLoS One. 2015;10(3):e0118241.

22. Medveczky T, Jackson M. Procalcitonin use in acute exacerbations of COPD. In: Paper presented at: European Respiratory Society Annual Congress; 2011 Sep 24-28; Amsterdam.

23. Nangia V, Gandhi K. Use of procalcitonin to guide the antibiotic therapy in patients with an acute exacerbation of COPD in a resource-limited setting: a case-control study. In: Paper presented at: 22nd European Congress of Clinical Microbiology and Infectious Diseases; 2012 Mar 31-Apr 3; London.

24. Sabrine N, Nejla T, Habiba S, et al. Procalcitonin guided antibiotic therapy during severe acute exacerbation of chronic obstructive pulmonary disease requiring mechanical ventilation: a before after study. Paper presented at: French Intensive Care Society, International Congress; 2018 Jan 24-26; Paris.

25. Townsend J, Adams V, Galiatsatos P, et al. Procalcitonin-guided antibiotic therapy reduces antibiotic use for lower respiratory tract infections in a United States medical center: results of a clinical trial. Open Forum Infect Dis. 2018;5(12):ofy327.

26. FDA clears test to help manage antibiotic treatment for lower respiratory tract infections and sepsis [Internet]. Washington (D.C.): U.S. Food \& Drug Administration; 2017. https://www.fda.gov/ NewsEvents/Newsroom/PressAnnouncements/ ucm543160.htm.

27. Rohde GG, Koch A, Welte T. Randomized double blind placebo-controlled study to demonstrate that antibiotics are not needed in moderate acute exacerbations of COPD-the ABACOPD study. BMC Pulm Med. 2015;15:5.

28. Loebinger MR, Shoemark A, Berry M, et al. Procalcitonin in stable and unstable patients with bronchiectasis. Chron Respir Dis. 2008;5(3):155-60.

29. Covington EW, Roberts MZ, Dong J. Procalcitonin monitoring as a guide for antimicrobial therapy: a review of current literature. Pharmacotherapy. 2018;38(5):569-81.

30. Soler N, Esperatti M, Ewig S, et al. Sputum purulence-guided antibiotic use in hospitalised patients with exacerbations of COPD. Eur Respir J. 2012;40(6):1344-53.

31. Daniels JM, de Graaff CS, Vlaspolder F, et al. Sputum colour reported by patients is not a reliable marker of the presence of bacteria in acute 
exacerbations of chronic obstructive pulmonary disease. Clin Microbiol Infect. 2010;16(6):583-8.

32. Llor C, Moragas A, Hernandez S, et al. Efficacy of antibiotic therapy for acute exacerbations of mild to moderate chronic obstructive pulmonary disease. Am J Respir Crit Care Med. 2012;186(8): 716-23.

33. Colak A, Yilmaz C, Toprak B, et al. Procalcitonin and CRP as biomarkers in discrimination of community-acquired pneumonia and exacerbation of COPD. J Med Biochem. 2017;36(2):122-6.

34. Falsey AR, Becker KL, Swinburne AJ, et al. Utility of serum procalcitonin values in patients with acute exacerbations of chronic obstructive pulmonary disease: a cautionary note. Int J Chron Obstruct Pulmon Dis. 2012;7:127-35.

35. Grolimund E, Kutz A, Marlowe RJ, et al. Long-term prognosis in COPD exacerbation: role of biomarkers, clinical variables and exacerbation type. COPD. 2015;12(3):295-305.
36. Huerta A, Crisafulli E, Menendez R, et al. Pneumonic and nonpneumonic exacerbations of COPD: inflammatory response and clinical characteristics. Chest. 2013;144(4):1134-42.

37. Lacoma A, Prat C, Andreo F, et al. Value of procalcitonin, C-reactive protein, and neopterin in exacerbations of chronic obstructive pulmonary disease. Int J Chron Obstruct Pulmon Dis. 2011;6:157-69.

38. Pizzini A, Lunger F, Sahanic A, et al. Diagnostic and prognostic value of inflammatory parameters including neopterin in the setting of pneumonia, COPD, and acute exacerbations. COPD. 2017;14(3): 298-303.

39. Metlay JP, Waterer GW, Long AC, et al. Diagnosis and treatment of adults with community-acquired pneumonia. Am J Respir Crit Care Med. 2019;200(7):e45-e67.

40. Bun RS, Scheer J, Guillo S, et al. Meta-analyses frequently pooled different study types together: a meta-epidemiological study. J Clin Epidemiol. 2020;118:18-28. 